\title{
An Association Rule Mining for Materialized View Selection and View Maintenance
}

\author{
P.R. Vishwanath \\ Associate Professor \\ CSE, RITS \\ Hyderabad
}

\author{
Rajyalakshmi \\ Professor \\ CSE,JNTUK \\ Vizaynagaram
}

\author{
Sridhar Reddy \\ Professor \\ Principal,SCET \\ Hyderabad
}

\begin{abstract}
Data warehouse (DW) is a repository with query interface in support of Decision support systems. DW required answering many complex queries, managerial level queries and analytical queries, needing to develop advanced computing techniques. The DW system process involving data modeling, ETL process, query interface and reporting system. Materialized views (MV) are the pre calculated views which are used to increase the DW system performance. MV selection and maintenance need to adopt new trends and techniques. Data mining (DM) is the process of extracting hidden useful information from huge data bases .Literature of Data Mining (DM) involving algorithms and techniques related to association, classification and clustering. Recent researches shown Data mining can also be used in optimization of calculating MV's. In addition to DM techniques are also used in efficient calculation of Data cubes. This paper proposed frequent rule mining on of the Data mining approach for the selection and maintenance of MV'S. By using the advanced concepts of frequent mining algorithm the query response time can be decreased. The approach also combines the advanced techniques to accommodate the changes in updating the base data so as to increase the performance of existing MV'S selection and maintenance approaches.
\end{abstract}

\section{Keywords}

Data warehouse, Data mining, Data cubes, DW Maintenance, Clustering.

\section{INTRODUCTION}

According to $\mathrm{W} \mathrm{H}$ Inman data warehouse is a subjectoriented, integrated, nonvolatile and Time-variant collection of data in support of management's decisions [1]. The tasks of data warehouse are data cleaning, data integration, data consolidation and summarization. Data warehouse uses the update driven approach rather than query driven approach in which data from multiple heterogeneous sources is integrated in advance and stored in a warehouse/repository for direct querying and analysis. Different Data warehouse tools are used at different levels such as data modeling tools, ETL tools, Multi-dimensional data base tools, reporting and analysis tools.

Data warehouse backend tools include function such as Data extraction, Data cleaning, Data transformation, Load and refresh. As the Data warehouse contains huge volumes of historical data OLAP technology demands the queries to be answered in span of seconds. It requires efficient multidimensional models such as cube technology, access methods and query processing techniques to decrease the work load on Data warehouse system. This paper uses partial materialization to decrease the storage and quick response time.
Data mining refers to extracting hidden useful knowledge from large amounts of data. The tasks of data mining are characterization, association, classification, and clustering and outlier analysis. Data mining has become an important tool in information technology as huge amount of data is converted into useful information and knowledge. Number of algorithms and approaches on each task on data mining are developed these algorithms are used in different applications such as medical, clinical, banking and communication. These algorithms are also used in improving the performance of data warehouse (Data warehouse maintenance).

As the Data warehouse is the collection of integrated heterogeneous sources which are generally relational databases or flat files. Data Warehouse has to undergone different technical stages such as data modeling, ETL (Extraction, Transformation, Loading), MDBS (MultiDimensional Data Base), Query Interface and reporting. After the ETL process the data store is divided into data marts or into small data warehouses concept wise or fact wise. At this stage DW system is expected to answer the complex queries raised to support decision. Consider the set of SQL statements posed on DW system as workload. The schema of the Data warehouse contains Dimensions tables and fact table and this paper uses Star Schema which contains Fact table centered with its Dimension tables. Materialized view is a pre calculated derived relations which are stored in the database. Since it is not possible to calculate all MV's for each attribute of each dimension and for also each fact, Partial materialization can be considered as the appropriate solution for it. In this appropriate queries are selected to be pre calculated. It is difficult process to select some queries out of all views so this process uses the optimized techniques to decrease the views number. Data mining techniques such as Association, clustering, classification can be used to find appropriate MVS. This paper uses Association mining algorithm such as Apriori algorithm to get the rules to find frequent item set which in turn uses the concept to find frequent queries. In the given workload each query is the SQL statement and each query contains a set of attributes .This approach considers each query as one transaction and the attributes presented in the query are taken as items. Primary process involving in this paper is transforming the queries into transactional data bases then Apriori algorithm is used to find best associations and the transactions are transformed back into queries. The resultant queries are frequent queries which are the reduced workload of given workload or MV selection process. This workload is used to calculate materialized views which are ready to answer the most queries asked. There may be updations in the base tables which are also in query form where the attributes affected for each updating are recalculated using the updating process. 
The paper is organized as Follows: Section 2 explains about data warehouse, data mining and materialized views. Section 3 explains the existing algorithms and approaches to find materialized views and usage of the different algorithms used in MV selection and MV maintenance. Section 4 explains the approach, proposed algorithm, framework and architecture of the presented and proposed algorithms. Section 5 explains about Cost Function. Section $6 \& 7$ explains the experimental results and comparison with the existing algorithms. Section 8 explains conclusion and future enhancements.

\section{LITERATURE REVIEW 2.1 Data Warehouse}

According to Inman Definition Data warehouse is a subject oriented, time variant, nonvolatile and integrated collection of data in support of Decision support system [1]. Data warehouse technology includes data cleaning, data integration and online analytical processing. OLAP tools support multidimensional analysis and decision making. Relational data base model uses entity relationship model and schema contains set of entity and relationships between them. Popular Data Model for a data warehouse is multi-dimensional data model. Multi-dimensional data model is in the form of star schema, snow flake schema or a fact constellation schema. In the above most popular one is Star schema which contains central table known as fact table containing bulk of the data with redundancy and dimension tables for each direction.

\subsection{Data Mining}

Data mining refers to extracting hidden information or knowledge from large amount of data. Data mining (DM) is also known as knowledge discovery from data (KDD). KDD involving following steps 1) Data cleaning 2) Data integration3)Data selection4)data transformation 5)Data mining 6) Pattern evaluation 7)Knowledge presentation.

Data is input for the DM system and Interesting pattern is the output. Data mining functionality involve concept or class description. The data is associated with classes or concepts which is useful to describe classes and concepts in summarized concise and precise way which is achieved via data characterization data discrimination, Statistical measures and plots, OLAP rollup operations, Attribute oriented induction.

Mining frequent patterns, Associations and correlation: Mining frequent patterns involves finding a frequent item set, frequent occurring subsequence etc. Frequent item set mining involved finding Associations and correlations in data. The Data mining algorithms developed to find associations are Apriori algorithm, FP GROWTH algorithm etc.

Classification and prediction: Classification is the process of finding a model that describes and distinguishes data classes or concepts. The process involving finding decision tree or a set of rules or a neural network. Algorithms developed to find classifiers are ID3, C4.

Cluster Analysis: Clustering methodology in DM divides the given data into groups or cluster based on their similarity. Algorithms developed related to clustering are k-means, kmedoids etc.

Outlier Analysis: Generally some part of the data or data objects behavior do not comply with the model of the data finding such data is known as Outlier Analysis.
Evolutionary Analysis: Behavior of the data changes over time this type of data needs separate mechanism which is known as Evolution Analysis

\subsection{Materialized Views}

Materialized views comprise pre computed and summarized information with the aim of answering most queries posed on data warehouse thereby saving of query processing time and storage. With the increase of attributes in each dimension there is need of increase of pre calculation of MVs. In this regard there is the increase of work load, needs to decrease the response time and storage. There are many view selection algorithms proposed. Continuous updating in the base table is to be reflected in the dimension a table, as the information is in DW is in the form of Fact. It is not possible to change whole DW instead changes to be accommodated at only affected part of DW. For this updating many views maintenance algorithms are proposed.

\section{EXISTING APPROACHES}

Different approaches have been proposed for improving the performance Materialized View selection and View maintenance of the Data Warehouse using different Data Mining techniques such as association, clustering etc. [10] There are many algorithms proposed for MV selection and MV maintenance algorithm. The following are the research papers presented on materialized views based on data mining techniques. T. Nalini et al. [6] presented an algorithm with IMine for MVs in DW. This work proposes cost effective mechanism for Materialized selection. Query frequency, query processing cost and space requirement are considered to materialize the candidate views. T.Nalini et al. [3] Presented an algorithm with IM-LSI index for incremental maintenance of MV which makes use of DM technique I-MINE to find frequent quires to pre calculate as a part of MV's configuration also described the updating of the MV's when Updations are done in base table it makes use of version table and proposed a cluster based MV selection in DW. T.V.Vijaykumar et al. [8] proposed frequent queries identification for constructing materialized views. This paper has four stages of implementation they are Domain creation, frequent queries identification, optimal queries selection and optimal queries merging. In this similar subject related queries are grouped together to form Domain creation. In this Hierarchical clustering is used to find clusters. In the merging process, Jaccard's co-efficient is used as the similarity measure. In the frequent queries identification the data mining technique called frequent mining is used. After the above four stages, the process remains with set of materialized views to be stored. This procedure reduces the time and ready to answer future queries. Dong Xin et al. [7] proposed StarCubing: Computing Iceberg Cubes by Top-Down and Bottom-Up Integration. To implement Data warehouse, one of the model proposed is Multi Dimensional Data model (MDM). 3.5 Kamel Aouiche et al [5] proposed Clustering based materialized selection using the clustering, one of the data mining technique. This algorithm uses workload approach. This procedure uses a query clustering involving similarity and dissimilarity measures defined on the workload queries. Gang,Zhao et al. [4] proposed the CBDMVS algorithm( clustering based dynamic materialization view selection algorithm) which makes use of clustering technique to decrease the computational cost and space. Yogeshree D. Choudhari et al. [9] proposed the cluster based approach for selection of materialized views. The procedure uses the clustering of the views. This algorithm uses the record generator. 


\section{PROPOSED ALGORITHM}

In this approach the set of SQL statements considered as workload which is selected to configure materialized views for partial materialization. This approach of ARMMVVM has two parts materialized view selection (MVS) and Materialized view maintenance (MVM) algorithms. The first step is to transform set of SQL statements into transactional data base. Apriori rule mining algorithm is applied on the transactional database to get frequent Item sets. The transactions not holding the frequent sets are eliminated and the remaining transactions are then transformed to respective queries. The resultant queries are Partial MVs set to be calculated. Finally updation process is applied to these MVs to get MVs updated. Consider the attributes in the base relation are $\left\{a_{1}, a_{2}\right.$ $\left.\ldots a_{n}\right\}$.Consider the workload i.e. Set of SQL statements previously posed are $\left\{\mathrm{Q}_{1}, \mathrm{Q}_{2}, \mathrm{Q}_{3} \quad \ldots \mathrm{Q}_{\mathrm{r}}\right\} . \quad\left\{\mathrm{a}_{\mathrm{i} 1}, \mathrm{a}_{\mathrm{i} 2}\right.$, $\left.a_{i 3} \ldots \ldots \ldots . . a_{i j}\right\}$ are the attributes present in the query $Q_{i}$. Each $\mathrm{Q}_{\mathrm{i}}$ transformed to transaction $\mathrm{T}_{\mathrm{i}}$ by taking the attributes in it. The set of all $\mathrm{T}_{\mathrm{i}}$ 's form the transactional Database. Applying the MVS algorithm on this Transaction Data base we get reduced set of Ti's. This set is transformed to the set of $\mathrm{Q}_{i}{ }^{\text {'s }}$ which is the reduced work load.

In the query I such as SELECT sales.time_id, sum (quantity_sold), sum (amount_sold) from SALES, TIMES where sales.time_id = times.time_id and times.fiscal_year in $(\mathbf{1 9 9 8 , 2 0 0 0 )}$ group by sales.time_id . The transaction with respect to Qi is Ti represented as Ti: time_id, fiscal year. The Association mining called Apriori algorithm is used to find Association rules so as to get frequent item sets.

\subsection{Materialized View Selection (MVS):}

Step 1: $Q$ the set of queries $Q_{i}$

Step 2: Transform $Q_{i}$ to Transactions/Items $T_{i}$

Step 3: Apply Apriori algorithm on $T=\left\{T_{1}, T_{2}, \ldots T_{r}\right\}$ to get frequent Item Sets.

Step 4: Find the $T^{1}$ the set of transactions containing the frequent item sets.

\section{Step 5: Find $\mathrm{Q}^{1}$ from $\mathrm{T}^{1}$}

Step 6: $\mathrm{Q}^{1}$ is the required Selection of Materialized Views (partial) from Q (Total).

Apriori algorithm is applied on the transactional database $\mathrm{T}=$ $\left\{\mathrm{T}_{1}, \mathrm{~T}_{2}, \mathrm{~T}_{3} \ldots \ldots . \mathrm{T}_{\mathrm{r}}\right\}$ to get best association rules on this transactional data bases. Rule 1 , Rule $2 \ldots$ Rule $\mathrm{j}$ are the rules used find frequent item sets .Each rule finds the transactions holding these frequent item set .In this process the transactions not holding any frequent item set are eliminated. The transaction set $\left\{\mathrm{T}_{\mathrm{k} 1}, \mathrm{~T}_{\mathrm{k} 2} \ldots . \mathrm{T}_{\mathrm{kp}}\right\} \quad(1 \leq \mathrm{kp} \leq \mathrm{r})$ remained are the resultant transactional data base as the output on applying apprioi algorithm. Next to find the respective queries set $\left\{\mathrm{Q}_{\mathrm{k} 1}, \mathrm{Q}_{\mathrm{k} 2} \ldots . . \mathrm{Q}_{\mathrm{kp}}\right\} \quad$ from $\left\{\mathrm{T}_{\mathrm{k} 1}, \mathrm{~T}_{\mathrm{k} 2} \ldots \mathrm{T}_{\mathrm{kp}}\right\} \quad(1 \leq \mathrm{kp} \leq \mathrm{r})$. These queries are now calculated and considered for configured set of MVs. This enhanced Architecture deducted 27\% of the queries from the given work load of 61 Queries. Fig 1 shows the frame work for proposed work

The above ARMMVS algorithm reduces the workload. After the Selection of MV's by above algorithm there may be changes in the base relations due to the updations in base table. These updations are to be reflected in the views selected in above reduced workload. This paper presents the Updation process in addition to the above process as part of View Maintenance. Updation process is used to the configured workload. Each Updation is recorded as one query. These
Queries are transformed as transaction $\mathrm{TD}_{\mathrm{i}}$. Each $\mathrm{TD}_{\mathrm{i}}$ contains attributes as in ARMVS algorithm.

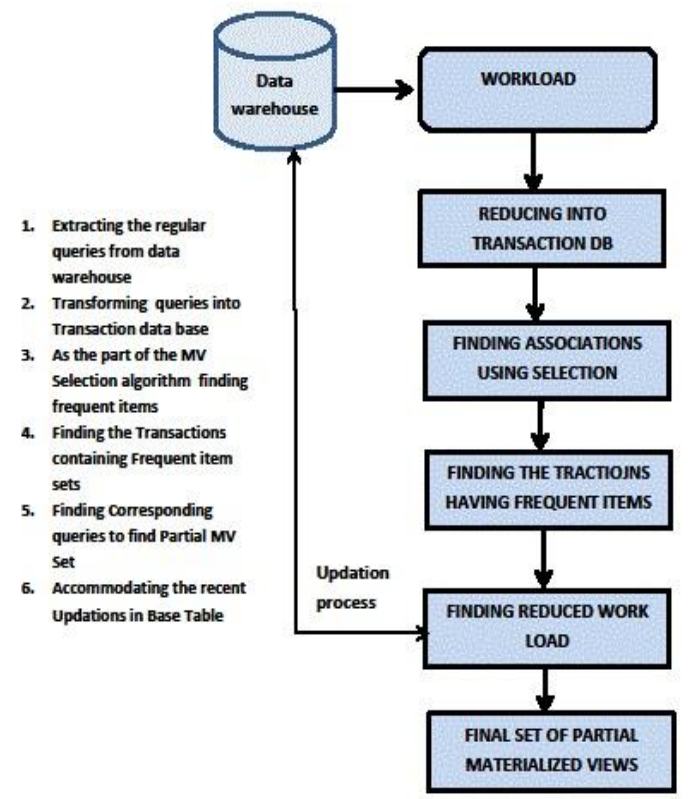

Fig1: Frame work for MVS and MVM

\subsection{Materialized View Maintenance (MVS):}

$\mathrm{Q}_{\mathrm{i}}$ : The query containing some or all attributes from set of attributes from $\left\{a_{1} \cdot a_{2} \ldots \ldots a_{n}\right\}$.

$\mathrm{T}_{\mathrm{i}}$ : The set of attributes $\left\{\mathrm{a}_{\mathrm{ij}}\right\}$ Corresponding to $\mathrm{Q}_{\mathrm{i}}$ for $\mathrm{i}=1$, $2, \ldots$.r. and $a_{i j} \in\left\{a_{s}\right\}$ for some $1 \leq \mathrm{s} \leq \mathrm{n}$ and for some $1 \leq \mathrm{j} \leq \mathrm{n}$

i.eT $T_{1}=\left\{a_{11}, a_{12}, \ldots . a_{1 s}\right\}, T i=\left\{a_{i 1}, a_{i 2}, \ldots a_{\mathrm{ij}}\right\}$

D: The set of all updated queries.

TD: The set of all attributes Contained in queries in D

$\mathrm{TD}=\left\{\mathrm{TD}_{1}, \mathrm{TD}_{2}, \ldots . . \mathrm{TD}_{\mathrm{t}}\right\}$ where $\mathrm{TD}_{\mathrm{x}}=\left\{\mathrm{a}_{\mathrm{p}}\right\}$ for Some $\mathrm{p}(1 \leq$ $\mathrm{p} \leq \mathrm{n})$

Q: The set of reduced work load by MVS algorithm

$\mathrm{T}^{\mathrm{l}}$ : The set of Transactions corresponding to $\mathrm{Q}^{\mathrm{l}}$

We construct $\mathrm{DT}^{\mathrm{l}}$ using step2 of following algorithm

Step1: Input $\mathrm{T}^{1}$ and $\mathrm{TD}_{\mathrm{x}}$

Step2: For $\mathrm{i}=1,2 \ldots r, \mathrm{a}_{\mathrm{ij}} \in \mathrm{Ti}, \mathrm{TD}_{\mathrm{x}} \in \mathrm{TD}$

$$
\left\{\begin{array}{l}
\text { Ti } \in D T^{1} \text { if } a_{i j} \in T^{x} \text { for } 1 \leq x \leq t \text { and } \\
\text { for } 1 \leq j \leq n \\
\text { Ti } \notin D T^{1} \text { otherwise. }
\end{array}\right.
$$

Step3: $\mathrm{DT}^{\mathrm{l}}$ is the set of transactions from $\mathrm{T}^{\mathrm{l}}$. Corresponding to each element in $\mathrm{DT}^{1}$ we get a Query in $\mathrm{Q}^{1}$. Therefore $\mathrm{DQ}^{1}$ is the set of Queries which is a part of $Q^{1}$

Step4: $\mathrm{DQ}^{1}$ is the set of queries to be pre-calculated from $\mathrm{Q}^{1}$.

In the above MVM algorithm, set of transactions $\mathrm{TD}_{\mathrm{i}}$ considered as TD. Further each transaction in TD is compared with the each transaction in the $\mathrm{T}^{\mathrm{l}}$ of MVS algorithm. By MVM algorithm we get $\mathrm{DT}^{1}$ which the subset of $\mathrm{T}^{1}$ of MVS 
algorithm. Finally the MVM algorithm gives the output $\mathrm{DQ}^{1}$ which is the subset of $\mathrm{Q}^{1}$ of MVS algorithm. This DQ1 required to be recalculated instead of recalculating all the queries in $\mathrm{Q}^{1}$ with the effect of updation of values of attributes in base tables.

\section{COST MODEL}

We use the cost model in which the cost of answering the query is assumed to be equal to the number of tuples in the aggregate used to answer the query. Our experiments show that there is a linear ship between sizes and running time of query. The cost of answering a query q is equal to the number tuples read to return the answer. We introduced THE GAIN MEASURE (GM) to measure the gain from work load to the resultant queries.

\subsection{The Gain Measure}

The gain measure of an aggregate view $s$ is computed by adding up the saving in query cost for each view $t$, including $\boldsymbol{s}$, over answering from the base view. If a set $\boldsymbol{D}$ of aggregated views is chosen for materialization, the gain of $\boldsymbol{D}$ is the sum of the gain of all views in $\boldsymbol{D}$. We now calculate the gain of an aggregate view. If $\boldsymbol{D}$ is a set of aggregates that have already been selected for pre computation, the gain of views is concerned with how materializing $s$ improves the cost of computing other views, including it. Let $\boldsymbol{C}(\boldsymbol{s})$ be the cost of computing another view from $\mathbf{s}$. looking back to our cost model $\boldsymbol{C}(\boldsymbol{s})$, is the number of tuples in $\boldsymbol{s}$. The gain of $\mathbf{s}$ with respect to the $\boldsymbol{D} . \boldsymbol{G}(\boldsymbol{s}, \boldsymbol{D})$ is defined as follows

1. For each aggregate view $s \leq \boldsymbol{t}, \boldsymbol{G}(\boldsymbol{s})$ defined as

a) Let $\boldsymbol{r}$ be the least cost view in $\boldsymbol{D}$ such that $\boldsymbol{s} \leq \boldsymbol{r}$

b) If $\boldsymbol{C}(\boldsymbol{t})<\boldsymbol{C}(\boldsymbol{r})$ the $\boldsymbol{G}(\boldsymbol{s})=\boldsymbol{C}(\boldsymbol{r})-\boldsymbol{C}(\boldsymbol{t})$,else $\boldsymbol{G}(\boldsymbol{s})=$ 0

\section{2. $\boldsymbol{G}(\boldsymbol{s}, \boldsymbol{D})=\sum_{\boldsymbol{s}<t} \boldsymbol{B}(\boldsymbol{s})$}

Precisely, for each view $\mathrm{s}$ that is a descendant of $\boldsymbol{t}$, we check to see if computing $\mathrm{s}$ from $s$ is cheaper that computing $\mathrm{s}$ from any other view in the set $\boldsymbol{D}$. If this is the case, then pre computing $\boldsymbol{t}$ gains $\boldsymbol{s}$. Since all aggregates can be computed from the (un-aggregated) base data, in step 1(a) we can always find a least cost aggregate view $\boldsymbol{r}$. Using Gain Measure two approaches ARMMVVM and CBMVS are compared and results are presented in fig 6 .

\section{EXPERIMENT AND RESULTS}

In order to verify proposed framework for MV selection and maintenance use of the work load containing sixty one data warehouse queries is required. The queries taken from http://eric.univ-lyon2.fr/ kaouiche/adbis.pdf.Tests are carried on up to $2 \mathrm{~GB}$ data warehouse using the Data base oracle .A system with configuration Pentium Core to Duo PC with 2 GB RAM and a 500GB hard disk. This Data Warehouse contains five dimension table and one fact tables. Dimension tables are PRODUCTS, TIMES, CHANNELS, PROMOTIONS, CUSTOMERS.PRODUCTS table containing 15 attributes, CHANNELS table containing 4 attributes, PROMOTIONS table containing 8 attributes, CUSTOMERS table containing 15 attributes and TIMES table containing 31 attributes. FACT table is SALES. SALES containing quantity sold, amount sold as two facts.

Table 1 show the Dimension tables used from the Popular Schema known as Star Schema.
Table 1: Relations used in Example

\begin{tabular}{|c|l|l|}
\hline SNO & DIMENSION & ATTRIBUTE \\
\hline \multirow{3}{*}{1} & \multirow{3}{*}{ CHANNEL } & CHANNEL_ID \\
\cline { 3 - 3 } & & CHANNEL_DESC \\
\cline { 3 - 3 } & & CHANNEL_CLASS \\
\cline { 3 - 3 } & & CHANNEL_TOTAL \\
\hline
\end{tabular}

\begin{tabular}{|c|l|l|}
\hline SNO & DIMENSION & ATTRIBUTE \\
\hline \multirow{5}{*}{2} & \multirow{4}{*}{ PROMOTIONS } & PROMO_ID \\
& & PROMO_NAME \\
& & PROMO_SUBCATEGORY \\
\cline { 3 - 3 } & & PROMO_CATEGORY \\
& & PROMO_COST \\
& & PROMO_BEGIN_DATE \\
\cline { 3 - 3 } & & PROMO_END_DATE \\
\cline { 3 - 4 } & & PROMO_TOTAL \\
\hline
\end{tabular}

\begin{tabular}{|c|c|c|}
\hline SNO & DIMENSION & ATTRIBUTE \\
\hline \multirow{16}{*}{3} & \multirow{16}{*}{ CUSTOMER } & CUST_ID \\
\hline & & CUST_FIRST_NAME \\
\hline & & CUST_LAST_NAME \\
\hline & & CUST_GENDER \\
\hline & & CUST_YEAR_OF_BIRTH \\
\hline & & CUST_MARITAL_STATUS \\
\hline & & CUST_STREET_ADDRESS \\
\hline & & CUST_POSTAL_CODE \\
\hline & & CUST_CITY \\
\hline & & CUST_STATE_PROVINCE \\
\hline & & CUST_MAIN_PHONE_NUMBER \\
\hline & & CUST_INCOME_LEVEL \\
\hline & & CUST_CREDIT_LIMIT \\
\hline & & CUST_EMAIL \\
\hline & & CUST_TOTAL \\
\hline & & CUST_YEAR_OF_BIRTH \\
\hline
\end{tabular}

\begin{tabular}{|c|l|l|}
\hline SNO & DIMENSION & ATTRIBUTE \\
\hline \multirow{4}{*}{4} & \multirow{3}{*}{ PRODUCT } & PROD_ID \\
\cline { 3 - 3 } & & PROD_NAME \\
\cline { 3 - 3 } & & PROD_DESC \\
\cline { 3 - 3 } & & PROD_SUBCATEGORY \\
\cline { 3 - 3 } & & PROD_SUBCAT_DESC \\
\hline
\end{tabular}




\begin{tabular}{|l|l|}
\hline & PROD_CATEGORY \\
\hline \multirow{5}{|}{} & PROD_CAT_DESC \\
\hline & PROD_WEIGHT_CLASS \\
\hline & PROD_UNIT_OF_MEASURE \\
\hline & PROD_PACK_SIZE \\
\hline SUPPLIER_ID \\
\hline & PROD_STATUS \\
\hline & PROD_LIST_PRICE \\
\hline & PROD_MIN_PRICE \\
\hline
\end{tabular}

The queries are first transformed in to the transactional Data bases. The attributes present in each query are identified as transactional items. Each Qi in the figure represents one corresponding transaction $\mathrm{Ti}$ in Transaction data base. Figure 3 shows the present Query transaction table. $D=\{T 1, T 2$ ,.....T60,T61\} are the given transaction database as input to the Apriori algorithm. The best rules are found by the Apriori algorithm and from them the frequent item sets found are

$\{$ PROMO_ID,PROMO_CATEGORY $\},\{$ PROMO_CATEGO RY, PROD_ID $\}, \quad\{C U S T$ ID, CUST_GENDER , $\{$ PROD_ID, PROD_CATEGORY\}. From these Frequent Item sets transactions eliminated. The remaining Transactional Data base is given by $\{\mathrm{T} 2, \mathrm{~T} 3$, T4,T5,T10,T10,T13,T16,T17,T18,T19,T21-T25,T29,T3350,T52,T54-T61\}. Then our workload reduced to $\{$ Q2,Q3,Q4 ,Q5,Q10 ,Q10,Q13,Q16，Q17,Q18,Q19,Q21Q25,Q29,Q33-Q50,Q52,Q54-Q61\}, which has 45 queries for calculating MV's. Finally the updated queries resulted changes in attribute values of base table are taken. These queries are then transformed to transactions set by considering their attributes present in each updated queries. The elements are compared with the above 45 Transactions of output of MVS algorithm. T2, T29, T52 are the transactions as the output of MVM algorithm. There by MVM algorithm concludes Q2, Q29, and Q52 which requires re-computation so as to reflect updated queries on Base tables. The experiment is continued by generating the Transactional database of $1 \mathrm{~GB}, 5 \mathrm{~GB}, 10 \mathrm{~GB}$, and $15 \mathrm{~GB}$. Applying ARMMVVM approach more gain is obtained than CBMVS approach.

\section{COMPARISIONS}

Fig 2 shows the number of queries taken and the output after applying presented ARMMVVM algorithm. First taken 61 queries and increased up to 200 queries. The chart shows the reduced workload in each case .As Fig 6, shown the efficiency of the approach increases with the increase of the work load.

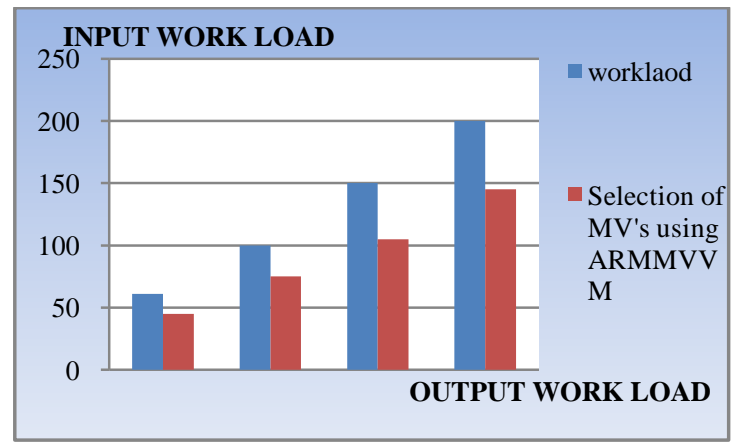

Fig 2: Reduced work load using ARMMVVM
Fig 3 shows the comparison between the CBMVS algorithm and ARMMVVM in each case by increasing the number of queries as in Fig 6. It is shown $10 \%$ to $20 \%$ reduced workload by using ARMMVVM algorithm. It shows that the ARMMVVM approach presented in the paper is better than CBMVS algorithm.

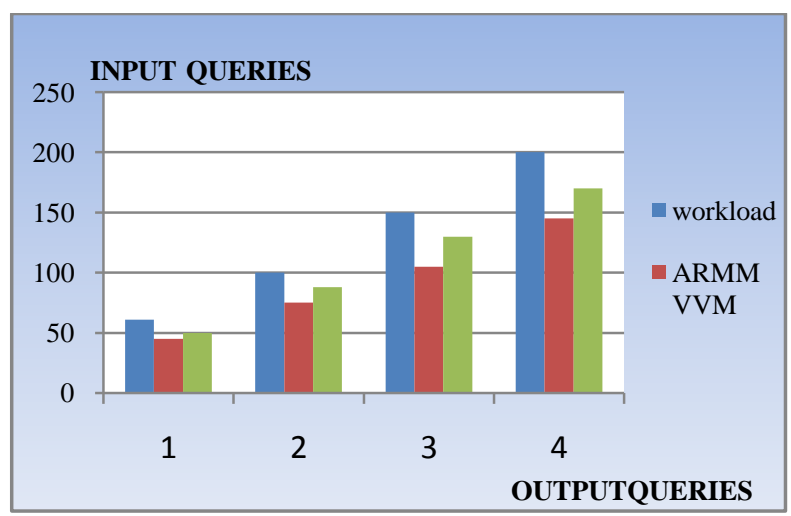

Fig 3: Comparison between ARMMVVM and CBMVS algorithm

Fig 4 shows the comparison between the CBMVS algorithm and ARMMVVM using the proposed GAIN MESURE. It is found by experiments that the Gain using the ARMMVVM is more than CBMVS algorithm.

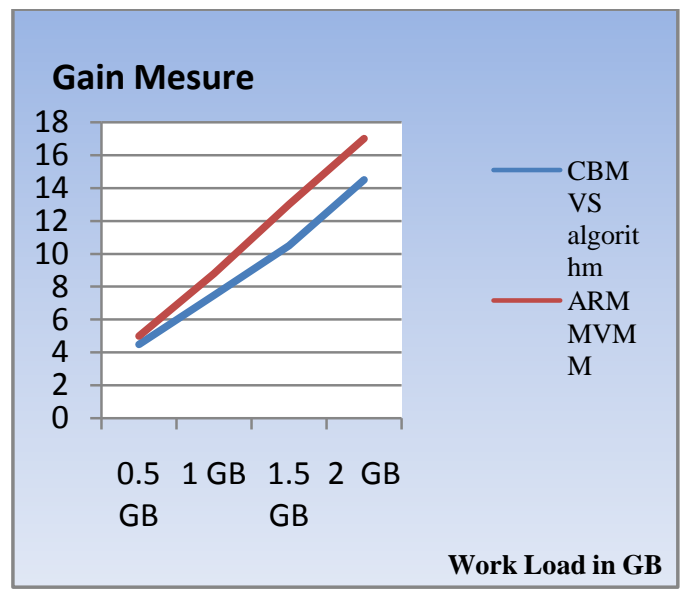

Fig 4: The comparison between ARMMVVM and CBMVS algorithm using Gain measure.

\section{CONCLUSION AND FUTURE WORK}

Data warehouse is the backend process for business analytics. With the dynamics in Analytics in reporting, the challenges are increasing day by day in Data ware house systems. Calculating the Appropriate MV's is the challenge in the DW research area. This paper presented the Association rule mining one of the Data mining technique used for improving the performance of the MV selection and MV maintenance. This Approach uses novel approach to find partial materialized views from the materialized views. Results of the approach show ARMMVVM is efficient when compared from the existing approaches and makes use of the data mining technique. This work can be extended by considering the fact table in rule mining for MV selection. Future work also extended to consider concept hierarchies for each Dimension. 


\section{REFERENCES}

[1] W. Inmon, "Building the data warehouse", Wiley publications, pp 23, 1991.

[2] Jiawei Han and MichelineKamber, "Data Mining Concepts and Techniques, $2^{\text {nd }}$ ed.Morgan Kaufmann Publishers, March 2006. ISBN 1-55860-901-6

[3] Nalini, T, Kumaravel, A and Rangarajan, K ,"A Novel Algorithm With Im-Lsi Index For Incremental Maintenance Of Materialized Views".Proceedings of JCS\&T Vol. 12 No. 1, April 2012

[4] An Gong, Weijing Zhao, "Clustering-based Dynamic Materialized View Selection Algorithm" Proceedings of Fifth International Conference on Fuzzy Systems and Knowledge Discovery, 2008, China,pp391-395

[5] K.Aouiche, P.EmmanuelJouve, and J.Darmont "Clustering-Based Materialized View Selection in Data Warehouses"TechnicalReport, University of Lyon 2, 2007.

[6] T.Nalini,Dr. A.Kumaravel, Dr.K.Rangarajan ," An Efficient I-Mine Algorithm For Materialized Views In A
Data Warehouse Environment", Ijcsi International Journal Of Computer ScienceIssues, Vol. 8, Issue 5, No 1, September 2011 Issn (Online):1694-0814

[7] D. Xin, J.Han, X. Li, and B.W.Wah. Star-cubing: Computing iceberg cubes by top-downand bottom-up integration. In Proc. 2003 Int. Conf. Very Large Data Bases (VLDB'03),Berlin, Germany, pages 476-487, Sept. 2003.

[8] T.V.Vijaykumar and KalyaniDevi,"Frequent queries identification for constructing materialized views",Journal of Computer Science \& Technology (JCS\&T);2012, Vol. 12 Issue 1s, p32.

[9] Yogeshree D. Choudhari,Dr. S. K. Shrivastava "Cluster Based Approach for Selection of Materialized Views " proceedings of International Journal of Advanced Research in Computer Science and Software Engineering (IJARCSSE)july2012

[10] P.R.Vishwanath Dr.D.Rajyalakshmi Dr.Sreedhar reddy "A Comparative Study of Data Mining Techniques in Maintenance of Data “, IJSER Volume 4, Issue 11, November 2013 Edition 